\title{
Editorial \\ CARIES DE LA PRIMERA INFANCIA: \\ La Declaración de Bangkok de la IAPD
}

\section{La 1 | INTRODUCCIÓN}

El propósito de esta declaración es obtener apoyo mundial para una definición basada en evidencia dela Caries de Primera Infancia (Early Childhood Caries - ECC), además de lograr un consenso en la comprensión de la etiología, factores de riesgo e intervenciones para reducir ECC, todas también basadas en evidencia. También presentará políticas y enfoques colaborativos para disminuir esta enfermedad crónica. Con estos antecedentes, 11 expertos de todo el mundo se reunieron bajoel auspiciodela Asociación Internacional de Odontopediatría (IAPD) para formular esta declaración.

\section{2 | LA DECLARACIÓN DE BANGKOK DE LA IAPD}

Caries de Primera Infancia (CPI) es definida como la presencia de una o más superficies cariadas (cavitadas o no cavitadas), perdida u obturada (debido a caries), en cualquier diente primario de un niño menor de 6 años. Los dientes primarios mantienen el espacio para los dientes permanentes y son esenciales para el bienestar del niño, debido a que la caries dental puede llevar a dolor crónico, infecciones y otras morbilidades. La Caries de Primera Infancia es prevenible, pero actualmente afecta a más de 600 millones de niños en el mundo, y permanece mayormente no tratada. Esta enfermedad tiene un gran impacto en la calidad de vida de los niños y sus familias y es una carga innecesaria a la sociedad.

La Caries de Primera Infancia, como otras formas de Caries, es considerada una enfermedad dinámica, mediada por la biopelícula, impulsada por azúcares, multifactorial, que resulta en un desbalance en la desmineralización y remineralización de los tejidos duros dentales.

La Caries Dental es determinada por factores biológicos, conductuales y psicológicos que están relacionados al medio ambiente del individuo. ECC comparte factores de riesgo con otras enfermedades no transmisibles (ENT) asociadas con consumo excesivo de azúcar, como enfermedades cardiovasculares, diabetes y obesidad. El exceso en la ingesta de azúcares lleva a una producción prolongada de ácidos de parte de bacterias que se adhieren a los dientes y a un cambio en la composición de la microbiota oral y el $\mathrm{pH}$ de la biopelícula. Si esto se mantiene, las estructuras dentarias se desmineralizan. ECC, en algunos casos está asociado a defectos del desarrollo del esmalte. El manejo apropiado de ECC por padres, profesionales de la salud y trabajadores de salud comunitarios bien informados, es importante para reducir la carga de esta enfermedad prevenible. La determinación del riesgo de Caries es una gran ayuda en esteprocesoyaqueestablecela probabilidad de que los pacientes individuales, o grupos de niños desarrollen lesiones cariosas. Para el niño individual, la determinación 
del riesgo es un elemento esencial para guiar en la prevención y el manejo. A nivel comunitario, la determinación del riesgo de caries puede guiar al diseño de intervenciones de salud pública y asignar el tiempo y los recursos hacia aquellos con mayores necesidades.

La prevención y el cuidado de ECC pueden ser estructurados en tres fases: Prevención Primaria que incluye mejorar la educación en salud oral para padres/cuidadores y trabajadores de la salud, limitar el consumo de azucares libres en comidas y bebidas, y exponer diariamente a los fluoruros. La Prevención Secundaria consiste en el control efectivo de las lesiones iniciales antes de su cavitación e incluye la aplicación de barnices fluorados con mayor frecuencia y la aplicación de sellantes de fosas y fisuras en molares susceptibles. La Prevención Terciaria, incluye la detención de las lesiones cavitadas y el tratamiento operatorio orientado a la preservación de la mayor cantidad de estructura dentaria.

\section{3 | RECOMENDACIONES}

Para reducir la prevalencia y la carga de ECC en el mundo, la declaración de Bangkok de la IAPD recomienda las siguientes acciones: Cuatro áreas clave requieren acciones de parte de las múltiples partes interesadas:

1. Concientizar a los padres / cuidadores, dentistas, higienistas dentales, médicos, enfermeras, profesionales de la salud, y otras partes interesadas acerca de ECC.

2. Limitar el consumo de azúcar en alimentos y bebidas y evitar los azúcares libres en niños menores de 2 años.

3. Realizar el cepillado dental dos veces al día con pasta dental fluorada. (al menos 1000 ppm) en todos los niños, utilizando una cantidad de pasta apropiada para la edad

4. Brindar guías preventivas durante el primer año de vida por medio de un profesional de la salud o un trabajador de salud comunitaria (a través de un programa existente - por ejemplo, vacunación - donde sea posible) e idealmente referir a un odontólogo para un cuidado continuo integral.

Adicionalmente se recomienda que:

- Las partes interesadas deben promover sistemas de reembolso y reformas educativas que enfaticen en prevención y manejo integral de ECC basados en evidencia.

- Para poder estandarizar a través de países y regiones, los estudios epidemiológicos deben considerar la presencia de lesiones cariosas cavitadas y no cavitadas; idealmente se debe reportar los estadios inicial, moderado y extenso de caries dental; los niños deben ser estudiados a los $3 y$ 5 años para determinar sus necesidades preventivas y restaurativas.

- Un currículo educativo sobre ECC debe ser implementado en las facultades y escuelas de Odontología de todo el mundo para asegurarse que al cuidado preventivo basado en evidencia - y 
en riesgo-, se le da igual peso que al manejo quirúrgico tradicional.

- Se deben apoyar investigaciones sobre inequidades en ECC, calidad de vida relacionado a salud oral, y economía dental, para poder entender el beneficio del cuidado efectivo y a tiempo.

El Apéndice que se muestra a continuación, preparado por el Panel de Expertos, brinda una Nota Comunicativa sobre Caries de Primera Infancia, diseñado para un variado grupo de profesionales y otras partes interesadas. Un articulo detallado titulado en inglés: 'Global Perspective of Early Childhood Caries Epidemiology, Aetiology, Risk Assessment, Societal Burden, Management, Education and Policy', brinda la evidencia más actualizada y las referencias que forman parte de esta declaración.

${ }^{*}$ La Cumbre Global sobre Caries de Primera Infancia se llevó a cabo en Bangkok, Tailandia del 2 al 4 de noviembre del 2018, Los miembros del Panel de Expertos que preparó esta declaración con aportes del Board de la IAPD fueron: Drs. N.B. Pitts (U.K), R. Baez (USA), C.Diaz-Guallory (USA), K. Donly (USA), C. Feldens (Brasil), C. McGrath (Hong Kong), P. Phantumvanit (Tailandia), K. Seow (Australia), N. Sharkov (Bulgaria), N. Tinanoff (USA), y S. Twetman (Dinamarca).

Los miembros del Board en Orden alfabético: Drs. M. Bonecker (Brasil), A. O'Connell (Irlanda), B. Drummond (Nueva Zelanda), T. Fujiwara (Japón), C. Hughes (USA), N. Kramer (Alemania), A. Kupietzky (Israel), A.M. Vierrou (Grecia), A. Tsai (Taiwan).

\section{REFERENCIA}

1. Tinanoff, N, Baez, RJ Diaz-Guillory, C, et al. Early childhood caries epidemiology, aetiology, risk assessment, societal burden, management, education, and policy: Global perspective. Int J Paediatr Dent. 2019;29:238-248. https://doi.org/10.1111/ ipd.12484

\section{APÉNDICE}

La Declaración de Bangkok de la IAPD: Nota Comunicativa sobre Caries de Primera Infancia

\section{¿Qué es Caries de Primera Infancia?}

- Caries Dental: Definición científicaCaries dental es una enfermedad dinámica, mediada por la biopelícula, impulsada por azúcares, multifactorial, que resulta en un desbalance en la desmineralización y remineralización de los tejidos duros dentales. La Caries Dental está determinada por factores biológicos, conductuales y psicológicos que están relacionados al medio ambiente del individuo.

- Caries de Infancia Temprana es: Definición corriente - Caries dental en niños pre-escolares que es común, mayormente no tratada y que tiene un profundo impacto en la vida de los niños.

Definición clínica - la presencia de una o más superficies cariadas (cavitadas o no cavitadas), perdida $\mathrm{u}$ obturada (debido a caries), en cualquier diente primario de un niño menor de 6 años. 
El contexto para Caries de Primera Infancia

- La Caries Dental es la enfermedad prevenible más común.

- La Caries Dental no tratada en dientes primarios afecta a más de 600 millones de niños en todo el mundo

- La Caries Dental comparte factores de riesgo común con otras enfermedades no transmisibles (ENT), asociadas con consumo excesivo de azúcares, como enfermedades cardiovasculares, diabetes y obesidad.

La carga inaceptable de Caries de Primera Infancia

- Caries de Primaria Infancia (ECC) es una carga inaceptable para niños, familias y la sociedad.

- La prevención y manejo de ECC apropiados y a tiempo son importantes para reducir esta carga y mejorar la calidad de vida de los niños globalmente.

¿Cómo podemos reducir Caries de Primera Infancia y su carga negativa?

- La Caries de Primaria Infancia (ECC) es multifactorial,ynohayunasoluciónúnica o fácil a este complejo "Rompecabeza de Caries". El compromiso de múltiples partes interesadas para abordar los diversos aspectos de las causas de Caries Dental es necesario para prevenir ECC.

\section{Prevención Primaria de ECC}

- Intervenciones sobre las causas primarias (río arriba) a nivel comunitario.
- Prevención de nueva enfermedad a nivel individual

\section{Prevención Secundaria de ECC}

- Control efectivo de lesiones iniciales antes de la cavitación.

- Detención de lesiones más avanzadas, cuando esto sea posible.

\section{Prevención Terciaria de ECC}

- Procedimientos de Control de Caries no invasivos.

- Cuidado restaurativo apropiado y preservando la estructura dentaria

Acción necesaria sobre ECC de parte de múltiples partes interesadas en cuatro áreas clave:

- Concientizar sobre ECC a los padres I cuidadores, dentistas, pediatras, enfermeras, otros profesionales de la salud, y otras partes interesadas

- Limitarla ingesta de azúcaren alimentos y bebidas y evitar los azúcares libres en niños menores de 2 años.

- Realizar el cepillado dental dos veces al día con pasta dental fluorada, (al menos 1000 ppm) en todos los niños, utilizando una cantidad de pasta apropiada para la edad

- Brindar guías preventivas durante el primer año de vida a través de un profesional de la salud o un trabajador de salud comunitaria (a través de un programa existente - por ejemplo, vacunación- donde sea posible) e idealmente referir a un odontólogo para cuidado continuo integral. 
Cómo citar este artículo:

Pitts, N, Baez, R, Diaz-Guallory, C, et al. Early Childhood Caries: IAPD Bangkok Declaration. Int J Paediatr Dent. 2019;29: 384-386.
Este artículo fue reproducido bajo licencia de autorización № 4665400630684 otorgada por John Wiley and Sons.

\section{Editorial \\ CÁRIE NA PRIMEIRA INFÂNCIA: Declaração de Bangkok da IAPD}

\section{1 | INTRODUÇÃO}

O objetivo desta Declaração é obter apoio em todo o mundo para uma definição baseada em evidências e uma compreensão sobre as evidências em relação à etiologia, fatores de risco e intervenções para reduzir Cárie na Primeira Infância (CPI), assim como estimular abordagens e políticas colaborativas para diminuir esta doença crônica. Neste contexto, 11 experts de todo o mundo foram convocados pela International Association of Paediatric Dentistry (IAPD) para criar este documento.

\section{2 | A DECLARAÇÃO DE BANGKOK DA IAPD}

Cárie na Primeira Infância (CPI) é definida como a presença de uma ou mais superfícies cariadas (cavitada ou não cavitada), perdidas ou restauradas (devido à cárie) em qualquer dente decíduo de uma criança com menos de seis anos de idade. Dentes decíduos mantêm o espaço para a dentição permanente e são essenciais para o bem-estar da criança, uma vez que cárie dentária na dentição decídua pode determinar dor crônica, infeções e outras morbidades. CPI é prevenível, mas atualmente afeta mais de 600 milhões de crianças no mundo, geralmente permanecendo não tratada. Esta doença tem um grande impacto na qualidade de vida de crianças e suas famílias e representa um impacto desnecessário para a sociedade.

Cárie na Primeira Infância, como outras formas de cárie, é considerada uma doença dinâmica multifatorial, determinada pelo consumo de açúcar e mediada por biofilme que resulta no desequilíbrio entre os processos de des e remineralização dos tecidos duros dentários. A cárie dentária é determinada por fatores biológicos, comportamentais e psicossociais relacionados ao meio do indivíduo. CPI compartilha fatores de risco comuns a outras doenças não transmissíveis (DNT) associadas ao consumo excessivo de açúcar, como doença cardiovascular, diabetes e obesidade. Consumo excessivo de açúcar determina produção prolongada de ácidos a partir de bactérias que se aderem ao dente e uma mudança na composição da microbiota oral e pH do biofilme. Se mantido, as estruturas do dente são desmineralizadas. CPI pode estar também as- 
sociada com defeitos de desenvolvimento do esmalte.

O manejo apropriado da CPI a partir da informação dos pais, profissionais da saúde e agentes comunitários em saúde, assim como políticas baseadas em evidências são importantes para reduzir o impacto desta doença prevenível. Avaliação do risco de cárie contribui neste processo por estabelecer a probabilidade de um paciente ou de um grupo de crianças desenvolver lesões de cárie. Em nível individual, avaliação de risco é um elemento essencial para guiar a prevenção e tratamento. Em nível coletivo, a avaliação do risco de cárie pode guiar intervenções públicas e alocar tempo e recursos para aqueles com maior necessidade.

A prevenção e tratamento de CPI podem ser estruturados em três fases. Prevenção primária inclui melhorar o conhecimento de pais/cuidadores e trabalhadores em saúde, limitando o consumo infantil de açúcares livres em bebidas e comidas e propiciando exposição diária ao flúor. Prevenção secundária consiste no controle efetivo de lesões iniciais antes da cavitação, o que pode incluir aplicações mais frequentes de verniz fluoretado e aplicação de selantes de fissuras em molares suscetíveis. Prevenção terciária inclui a paralisação de lesões cavitadas e tratamento operatório preservando a estrutura dentária.

\section{3 | RECOMENDAÇÕES}

Para reduzir a prevalência e impacto de CPI no mundo, a Declaração de Bangkok da IAPD recomenda as seguintes ações: quatro áreas essenciais descritas a seguir requerem ações de múltiplos atores:

1. Conscientizar pais/cuidadores, dentistas, técnicos em saúde bucal, médicos, enfermeiras, profissionais da saúde e outros grupos interessados sobre CPI.

2. Limitar o consumo de açúcar em alimentos e bebidas e evitar açúcares livres para crianças com menos de 2 anos de idade.

3. Escovar os dentes de todas as crianças duas vezes por dia com pasta fluoretada (ao menos 1000 ppm) usando uma quantidade adequada de dentifrício.

4. Prover orientações preventivas no primeiro ano de vida por um profissional da saúde ou agente comunitário de saúde (em conjunto com programas já existentes - p.ex. campanhas de vacinação - sempre que possível) e, idealmente, referir para um dentista para manutenção e cuidados preventivos.

Além disso, é recomendável que:

- Grupos interessados defendam sistemas de reembolso e uma reforma educacional que enfatizem a prevenção e o manejo de CPI baseados em evidências.

- Estudos epidemiológicos devem registrar a presença de lesões de cárie cavitadas e não cavitadas para padronizar comparações entre países e regiões; idealmente, devem ser registrados estágios iniciais, moderados e extensos de cárie; crianças devem ser avaliadas aos três e cinco anos de idade para que se verifiquem as necessidades preventivas e restauradoras. 
- Um currículo de educação em CPI deve ser implementado em Cursos de Odontologia no mundo para assegurar que cuidados preventivos baseados em evidências e no risco de cárie sejam ensinados da mesma forma que o tratamento cirúrgico tradicional.

- Pesquisas em desigualdades em CPI, qualidade de vida relacionada à saúde bucal, intervenções e economia em saúde devem ser apoiadas para permitir a compreensão sobre os benefícios do cuidado efetivo e oportuno.

O Apêndice abaixo, preparado pelo Grupo de Experts, apresenta uma Declaração sobre Cárie na Primeira Infância direcionada para vários profissionais e grupos interessados. Um artigo detalhado, intitulado "Global Perspective of Early Childhood Caries Epidemiology, Aetiology, Risk Assessment, Societal Burden, Management, Education and Policy"1 fornece evidências atualizadas e referências que basearam tal declaração.

* O Global Summit on Early Childhood Caries ocorreu em Bangkok de 2 a 4 de Novembro de 2018. Membros do Painel de Experts que desenvolveram esta Declaração com a contribuição do Comitê da IAPD foram: N. B. Pitts (Reino Unido), R. Baez (EUA), C. Diaz-Guallory (EUA), K. Donly (EUA), C. Feldens (Brasil), C. McGrath (Hong Kong), P. Phantumvanit (Tailândia), K. Seow (Austrália), N. Sharkov (Bulgária), N. Tinanoff (EUA) e S. Twetman (Dinamarca).

Membros do Comitê da IAPD em or- dem alfabética: M. Bönecker (Brasil), A. O'Connell (Irlanda), B. Drummong (Nova Zelândia), T. Fujiwara (Japão), C. Hughes (Estados Unidos). N. Krämer (Alemanha), A. Kupietzky (Israel), A.M Vierrou (Grécia), A. Tsai (Taiwan).

\section{REFERÊNCIA}

1. Tinanoff N, Baez R, Diaz-Guallory C, et al. Early Childhood Caries Epidemiology, Aetiology, Risk Assessment, Societal Burden, Management, Education, and Policy: Global Perspective. Int J Pediatr Dent. 2019; 29:238-248.

\section{APÊNDICE}

Declaração de Bangkok da IAPD sobre Cárie na Primeira Infância

\section{O que é Cárie na Primeira Infância (CPI)?}

- Cárie Dentária: Definição científica - Cárie dentária é uma doença dinâmica multifatorial, determinada pelo consumo de açúcar e mediada por biofilme que resulta no desequilíbrio entre os processos de des e remineralização dos tecidos duros dentários. Cárie dentária é determinada por fatores biológicos, comportamentais e psicossociais relacionados ao meio do indivíduo.

- Cárie na Primeira Infância - Definição leiga - Cárie dentária em crianças préescolares, uma doença comum, na maioria das vezes não tratada e que pode ter profundo impacto na vida das crianças. Definição clínica - presença de uma ou mais superfícies cariadas (cavitada ou não cavitada), perdidas ou restauradas 
(devido à cárie) em qualquer dente decíduo de uma criança com menos de seis anos de idade.

\section{$\mathrm{O}$ contexto de CPI}

- Cárie dentária é a doença prevenível mais comum.

- Cárie dentária não tratada em dentes decíduos afeta mais de 600 milhões de crianças no mundo.

- Cárie dentária compartilha fatores de risco comuns a outras doenças não transmissíveis (DNT) associadas com consumo excessivo de açúcar, como doença cardiovascular, diabetes e obesidade.

\section{O impacto inaceitável de CPI}

- CPI representa um impacto inaceitável para crianças, famílias e a sociedade.

- Prevenção e manejo oportuno e apropriado de CPI é importante para reduzir este impacto e melhorar a qualidade de vida de crianças no mundo.

\section{Como reduzir CPI e seu impacto?}

CPI é multifatorial e não há uma solução fácil e única para o complexo quebra-cabeças. O engajamento de múltiplos grupos interessados que levem em consideração os múltiplos aspectos da causa de cárie é necessário para prevenir CPI.

\section{Prevenção Primária em CPI}

- Intervenções amplas em nível da comunidade.

- Prevenção da doença em nível individual.

\section{Prevenção Secundária de CPI}

- Controle efetivo de lesões iniciais antes da cavitação.
- Paralisação de lesões mais avançadas, quando possível.

\section{Prevenção terciária de CPI}

- Procedimentos de controle de cárie não invasivos.

- Tratamento restaurador apropriado, preservando estrutura dentária.

Ações sobre CPI necessárias de múltiplos grupos interessados em quatro áreas chave

- Conscientizar pais/cuidadores, dentistas, técnicos em saúde bucal, pediatras, enfermeiras, outros profissionais da saúde e outros grupos interessados sobre CPI.

- Limitar o consumo de açúcar em alimentos e bebidas e evitar açúcares livres para crianças com menos de 2 anos de idade.

- Escovar os dentes de todas as crianças duas vezes por dia com pasta fluoretada (ao menos 1000 ppm) usando uma quantidade adequada de dentifrício.

- Prover orientações preventivas no primeiro ano de vida por um profissional da saúde ou agente comunitário de saúde (em conjunto com programas já existentes - p.ex. campanhas de vacinação - sempre que possível) e idealmente, referir para um dentista para manutenção e cuidados preventivos.

Como citar este artigo: Pitts N, Baez R, Diaz-Guallory C, et al. Early Childhood Caries: IAPD Bangkok Declaration. Int J Paediatr Dent. 2019; 29:384-386. 